\title{
Spatiotemporal analysis of the protein expression of angiogenic factors and their related receptors during folliculogenesis in rats with and without hormonal treatment
}

\author{
Dalhia Abramovich ${ }^{1}$, Alejandra Rodriguez Celin ${ }^{1}$, Fátima Hernandez ${ }^{1}$, Marta Tesone ${ }^{1,2}$ \\ and Fernanda Parborell ${ }^{1}$ \\ ${ }^{1}$ Instituto de Biología y Medicina Experimental (IBYME)-CONICET, Obligado 2490, 1428 Buenos Aires, Argentina and \\ ${ }^{2}$ Departamento de Química Biológica, Facultad de Ciencias Exactas y Naturales, Universidad de Buenos Aires, 1428 \\ Buenos Aires, Argentina
}

Correspondence should be addressed to F Parborell; Email: fparbo@dna.uba.ar

\begin{abstract}
This study investigated the protein expression and cellular localization of ANGPT1, ANGPT2, and their receptor TEK, as well as vascular endothelial growth factor A (VEGFA) and its receptor KDR (VEGFR2) during folliculogenesis. To obtain follicles at different stages for immunochemistry and western analyses, we used prepubertal untreated, diethylstilbestrol- and equine chorionic gonadotropin-treated rats. To confirm that these hormonal treatments reflect physiological change, we used non-treated adult rats. No expression of ANGPT1 was observed in granulosa cells (Gc) from immature hormone-treated and non-treated rats at any follicular stage. By contrast, ANGPT1 expression in theca cells (Tc) increased with follicular maturation. ANGPT2 protein was either absent or weakly expressed in Gc at all follicular stages. In Tc, minimal expression of ANGPT2 protein was detected in the preantral follicle (PF), whereas it was stronger in the early antral follicle (EAF) and preovulatory follicle (POF). TEK staining was absent in Gc but was intense in Tc at every follicular stage. Staining for VEGFA was either absent or weakly present in Gc and Tc in PF and EAF, although in POF it was stronger in Gc and Tc. Staining for KDR was absent in Gc and very low in Tc from PF. Gc and Tc of EAF showed positive staining for KDR and in POF the staining was stronger. These results were confirmed by western immunoblot. A similar pattern of expression of these proteins was observed in cycling rats. In conclusion, we observed that the protein expression of ANGPT1, ANGPT2, VEGFA and their receptors increased during follicular development in rats.
\end{abstract}

Reproduction (2009) 137 309-320

\section{Introduction}

Angiogenesis begins with capillary proliferation and culminates in the formation of a new microcirculatory bed composed of arterioles, capillaries, and venules. Many studies on angiogenesis and its regulation have focused on pathological processes because the regulation of the angiogenic process can become a novel treatment of various disorders (Folkman \& Klagsbrun 1987, Ferrara 1999, Pandya et al. 2006). The physiological angiogenesis in adults is rather limited, except for several aspects of reproduction, including cyclic changes in the ovary and reproductive tract, implantation, and placental function (Stouffer et al. 2001).

In the ovary, the vascular supply is formed on a cyclic basis. Non-growing primordial follicles and slowgrowing preantral follicles (PF) do not possess an independent capillary network and are dependent on their proximity to the stromal vessels. Soon after the antrum appears, the follicle acquires a vascular network in the theca layer that consists of two concentric networks of vessels in the theca interna and externa respectively. The selection of the dominant follicle may depend on both the differential formation of this rich vascular supply and an increase in vascular permeability (Zeleznik et al. 1981). On the contrary, one of the earliest signs of follicular atresia or degeneration is the association between the reduced DNA synthesis in endothelial cells and the reduced vascularity of the follicle (Greenwald 1989).

Vascular endothelial growth factor A (VEGFA) plays an important role in the regulation of angiogenesis in the ovary by acting through its receptor KDR (also known as FLK1 or VEGFR2; Shweiki et al. 1993, Klagsbrun \& D'Amore 1996, Geva \& Jaffe 2000). Expression of VEGFA in ovarian follicles depends on the follicular stage. In bovine, porcine, and murine ovaries, VEGFA is weakly expressed during early ovarian follicular development and its expression increases in granulosa and theca cells (Tc) throughout folliculogenesis (Barboni 
et al. 2000, Celik-Ozenci et al. 2003, Greenaway et al. 2004). However, protein expression and localization of the VEGFA receptor, KDR, in the rat ovary remains unknown.

While VEGFA is the main initiator of angiogenesis, the formation and differentiation of a structurally and functionally mature vascular network probably requires the coordinated action of various factors. These include the angiopoietins ANGPT1 and ANGPT2, which act via the tyrosine kinase receptor, TEK (Maisonpierre et al. 1997). Unlike VEGFA, ANGPT1 is unable to stimulate endothelial cell proliferation (Davis et al. 1996), but instead is required for the recruitment of peri-vascular cells that lead to the maturation and stabilization of newly developed capillaries (Suri et al. 1996, Maisonpierre et al. 1997). ANGPT1 is able to induce the phosphorylation of TEK, which subsequently transduces a biological effect. ANGPT2 binds to TEK with the same affinity as ANPGT1 but does not phosphorylate the receptor and, as a consequence, ANGPT2, is a natural antagonist of ANGPT1 (Maisonpierre et al. 1997). Previous studies demonstrate the expression of Vegfa, Angpt1 and Angpt2 mRNAs in the rat ovary (Phillips et al. 1990, Koos 1995, Maisonpierre et al. 1997), suggesting a role for these factors in ovarian angiogenesis. There are observations that ANGPT1 (but not
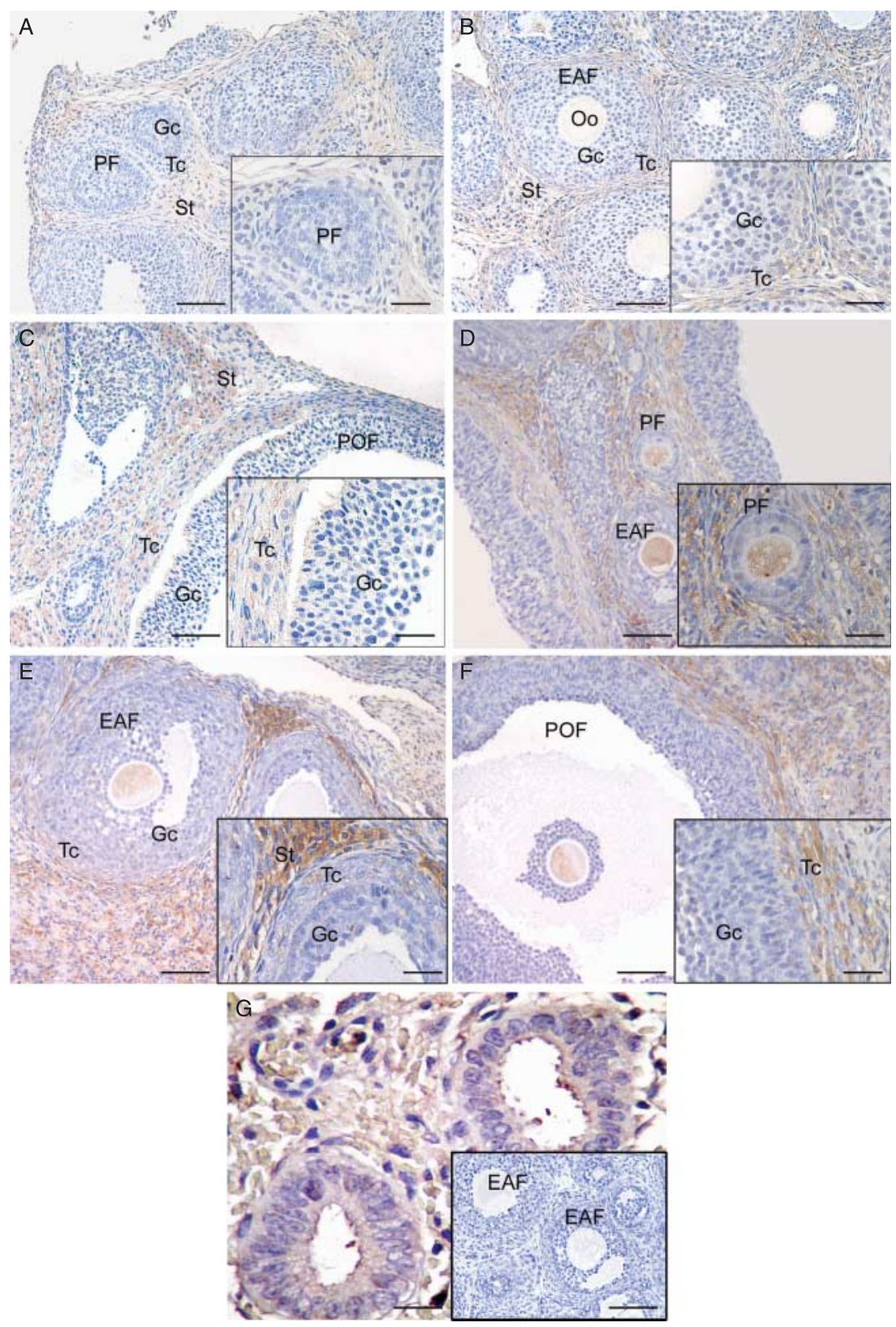

Figure 1 Immunohistochemical staining for ANGPT1 during follicular development in the rat ovary. (A) Untreated prepubertal rats. (B) DEStreated prepubertal rats. (C) eCG-treated prepubertal rats. D-F shows representative fields of ovarian sections from postpubertal cycling rats. (G) Human endometrium was used as a positive control and the glandular epithelium showed immunoreactivity for ANGPT1; inset: negative control. Specificity for ANGPT1 is supported by the loss of staining in follicular tissue preabsorbed with ANGPT1 peptide or in the absence of the primary antibody (not shown). PF, preantral follicle; EAF, early antral follicle; POF, preovulatory follicle; Gc, granulosa cells; Tc, theca cells; Oo, oocyte; St, stroma. Scale bars represent (A-F and negative control) $50 \mu \mathrm{m}$; (G, insets and positive control) $20 \mu \mathrm{m}$. 
ANGPT2) expression is elevated in luteinizing cells of the ovulatory follicle (Hazzard et al. 1999), and that ANGPT2 expression is associated with follicle atresia (Maisonpierre et al. 1997) and corpus luteum regression (Hazzard et al. 2000). Several studies have reported the expression of angiopoietins in the corpus luteum in cows, monkeys, and humans, suggesting that angiopoietins are involved in the regulation of corpus luteum development and luteal function via stabilization or destabilization of blood vessels in the corpus luteum (Hazzard et al. 2000, Wulff et al. 2000, Tanaka et al. 2004). Nevertheless, to date, there is little information about cell-type and time-specific expression of ANGPT1 and ANGPT2 proteins and their receptor TEK during folliculogenesis.

The acquisition of a suitable vascular contribution is probably a limiting step in the selection and maturation of the dominant follicle destined to ovulate. The development and growth of these capillaries are controlled by angiogenic factors produced by granulosa and Tc. These might act in different moments, some stimulating the growth and others promoting the reorganization of endothelial cells in more complex vascular structures. On the basis of these considerations, our hypothesis is that the expression of angiogenic factors as angiopoietins and VEGFA, as well as their receptors (TEK and KDR respectively), changes along with follicular development and, as a consequence, a more complex vascular network supplying tropic factors, hormone precursors, oxygen, and nutrients to the follicle is developed.

Therefore, the objective of the present work was to investigate the protein expression and immunohistochemical localization of ANGPT1, ANGPT2, their receptor TEK, and VEGFA and its receptor KDR, at different stages of rat follicular development. In order to characterize the follicular vascular network, we also analyzed blood vessels during follicular development.

\section{Results}

Figure 1 shows the immunohistochemical staining for ANGPT1 in ovarian sections from non-treated, diethylstilbestrol (DES)- or equine chorionic gonadotropin (eCG)-treated prepubertal rats and non-treated adult rats during the different follicular stages. In prepubertal rats, we found that ANGPT1 was not expressed in granulosa cells (Gc) at any follicular phase. By contrast, thecal cells (Tc) showed immunostaining for ANGPT1 at all follicular stages. In PF and early antral follicles (EAF), we detected minimal expression of ANGPT1 peptide (Fig. 1A and B). However, intense ANGPT1 immunostaining was observed in preovulatory follicles (POF; Fig. 1C). In postpubertal cycling rats, we observed a similar pattern of expression of ANGPT1 at each follicular stage when compared with the hormonally untreated and treated immature female rats (Fig. 1D-F).
Figure 2 shows expression of ANGPT1 in PF, EAF, and POF determined by western immunoblot. Expression of ANGPT1 was greater $(P<0.05)$ in EAF and POF than in PF.

Figure 3 shows the immunohistochemical staining for ANGPT2 in ovarian sections from non-treated, DES- or eCG-treated prepubertal rats and non-treated adult rats. In prepubertal rats, ANGPT2 protein was either absent or weakly expressed in Gc at all follicular stages. In Tc, ANGPT2 expression was greater in EAF and POF than in PF (Fig. 3A-C). In adult rats, we observed similar results for immunohistochemical staining for ANGPT2 during folliculogenesis when compared with immature rats (Fig. 3D-F).

Figure 4 shows expression of ANGPT2 protein determined by western immunoblot. Expression of ANGPT2 protein was greater $(P<0.05)$ in EAF and POF than in PF.

The ANGPT receptor TEK was immunodetected in ovarian sections from the different experimental groups during folliculogenesis (Fig. 5). In prepubertal rats, staining for TEK was absent in Gc at every follicular stage (Fig. 5A-C). Nevertheless, intense immunostaining in Tc for TEK was observed as the follicle developed. TEK-positive endothelial cells were identified by immunohistochemical staining for von Willebrand Factor and for TEK in adjacent paraffin sections (Fig. $5 \mathrm{G}-\mathrm{H}$ ). It is worth mentioning that endothelial cells were also stained for TEK in the follicle. In adult rats, we obtained a similar pattern of expression of TEK in each follicular stage when compared with that obtained for the immature female rats (Fig. 5D-F).

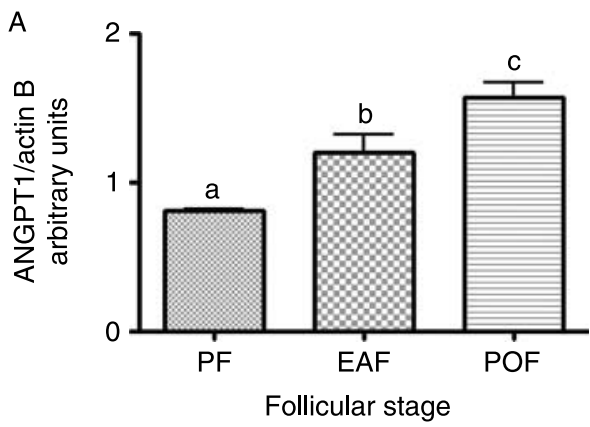

$B$

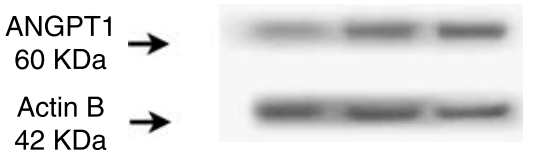

Figure 2 Expression analysis of ANGPT1 on follicular development in ovaries from prepubertal, DES-treated or superovulated rats. (A) Densitometric quantification of ANGPT1 content. (B) Representative immunoblot of ANGPT1. Bars represent the mean \pm s.E.M. normalized to $\beta$-actin. Different letters over the bars mean significant differences between groups $(n=8 ; P<0.05)$. 


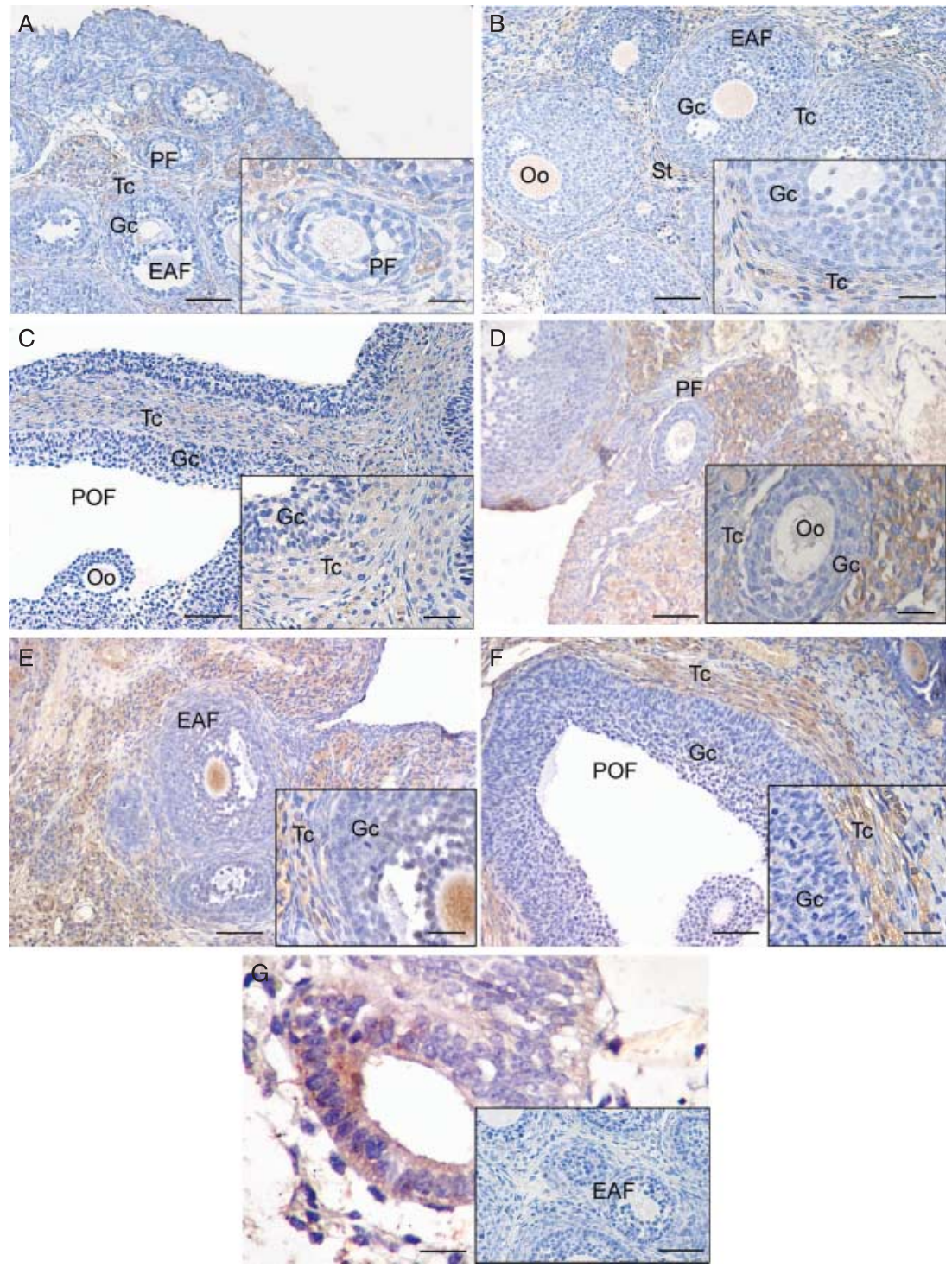

Figure 3 Immunohistochemical staining for ANGPT2 during follicular development in the rat ovary. (A) Untreated prepubertal rats. (B) DEStreated prepubertal rats. (C) eCG-treated prepubertal rats. D-F shows representative fields of ovarian sections from postpubertal cycling rats. (G) Human endometrium was used as a positive control and the glandular epithelium showed immunoreactivity for ANGPT2; inset: negative control. Specificity for ANGPT2 is supported by the loss staining in follicular tissue preabsorbed with ANGPT2 peptide or in the absence of the primary antibody (not shown). PF, preantral follicle; EAF, early antral follicle; POF, preovulatory follicle; Gc, granulosa cells; Tc, theca cells; Oo, oocyte; St, stroma. Scale bars represent (A-F and negative control) $50 \mu \mathrm{m}$; (G, insets and positive control) $20 \mu \mathrm{m}$.
Immunostaining of ANGPT1, ANGPT2, and their receptor, TEK was detected in the glandular epithelium of human endometrium, which was used as a positive control (Hirchenhain et al. 2003; Figs 1G, 3G, and 5I).

Figure 6 shows expression of TEK in PF, EAF, and POF by western immunoblot. The expression of TEK was the greatest $(P<0.05)$ in $\mathrm{POF}$, less in $\mathrm{EAF}$, and least in PF.

Figure 7 shows the presence and localization of VEGFA in the different stages of ovarian follicles. In immature rats, staining for VEGFA was either absent or weakly expressed in PF, Gc, and Tc (Fig. 7A). In EAF, staining for VEGFA was weak in both cellular types (Fig. 7B). POF exhibited moderate VEGFA immunoreactivity in Gc but intense in Tc (Fig. 7C).

Figure 8 shows the immunohistochemical staining for VEGFA receptor, KDR. In PF from immature rats, staining for KDR was absent in Gc and very low in Tc (Fig. 8A). Gc of EAF showed positive staining for KDR, being more intense in the Tc of these follicles (Fig. 8B). In POF, staining was stronger in Gc and Tc than in EAF (Fig. 8C). In postpubertal cycling rats, we observed a similar pattern of expression of VEGFA and its receptor KDR, in different developmental stages when compared with prepubertal rats (Figs 7D-F and 8D-F respectively).

Figure 9 shows expression of KDR protein in PF, EAF, and POF determined by western immunoblot. Expression of KDR protein was greater $(P<0.01)$ in EAF and POF than in PF.

Figure 10 summarizes the unequal distribution of the vasculature during follicular development. Primary and PF do not have their own blood vessels (Fig. 10A). As an antrum develops in the follicle to form EAF, the theca layer acquires a vascular sheath (Fig. 10B). This capillary 


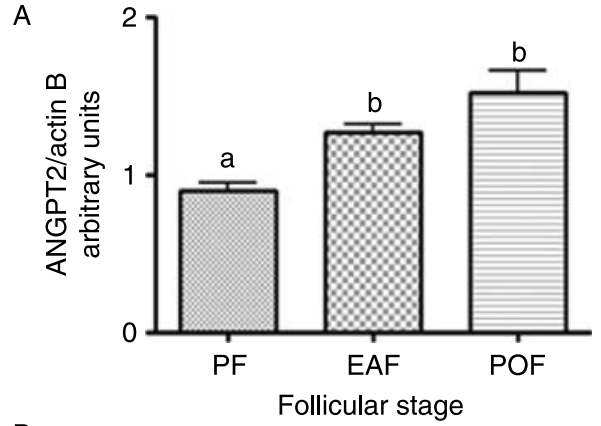

B

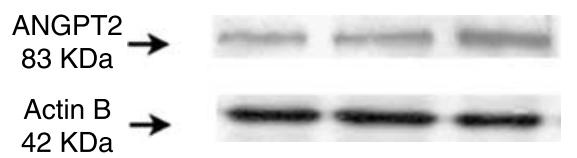

Figure 4 Analysis of the expression of ANGPT2 on follicular development in ovaries from prepubertal, DES-treated or superovulated rats. (A) Densitometric quantification of ANGPT2 content. (B) Representative immunoblot of ANGPT2. Bars represent the mean \pm S.E.M. normalized to $\beta$-actin. Different letters over the bars mean significant differences between groups $(n=8 ; P<0.05)$.

network continues developing as the follicle grows (Fig. 10C). By contrast, the granulosa layer remains avascular until ovulation.

\section{Discussion}

In the present study, we show protein expression levels and cellular localization of ANGPT1, ANGPT2, and their receptor TEK, as well as of VEGFA and its receptor $\mathrm{KDR}$, during follicular development in the rat ovary. In order to understand the role of these angiogenic factors in follicular growth, the development of the endothelial cell network was also determined.

We demonstrated by immunohistochemical studies and western immunoblot that the expression of ANGPT1 and ANGPT2 increased during follicular development and was mainly localized in Tc of follicles both in immature and adults rats. These findings are consistent with those reported by Maisonpierre et al. (1997) in in situ hybridization studies in rat ovaries, which show that Angpt 1 and Angpt2 mRNA is expressed in follicular cells during follicular development. Moreover, other studies performed in bovines and primates have shown that expression of Angpt1 mRNA increases during folliculogenesis (Hazzard et al. 1999, Stouffer et al. 2001, Shimizu et al. 2003). The gradual increase in ANGPT1 expression during folliculogenesis may lead to an increase in pericyte recruitment, thereby stabilizing the newly formed vascular network. This suggests that ANGPT1 protein might increase vascular stability by recruiting pericytes to developing capillaries (Suri et al. 1996, Hanahan 1997, Maisonpierre et al. 1997).
Angiopoietins represent one of the main modulators of angiogenesis by binding to their receptor TEK (Davis et al. 1996, Suri et al. 1996).

As shown in the present study, TEK immunoreactivity in Tc increased gradually throughout follicular development. However, TEK receptor immunostaining in Gc was absent in all follicular stages. The co-expression of ANGPTs and TEK receptor in Tc implies that the ANGPT system may exert both autocrine and paracrine actions.

It is worth to note that the changes observed in the different follicular stages induced by the hormonal treatments (DES or eCG) reflect the data obtained in ovaries from adult cycling rats, which suggest that our observations possess a physiological correlation and are not only the result of a hormonal treatment.

Our results are consistent with previous data showing the presence of Tek mRNA by in situ hybridization in bovine (Shimizu et al. 2003) and non-human primate ovaries (Stouffer et al. 2001) during follicular development. It is interesting to note that genetic manipulations in mice and cell-culture experiments suggest that ANGPT2 antagonizes ANGPT1 mediated by TEK binding (Suri et al. 1996, Maisonpierre et al. 1997, Scharpfenecker et al. 2005).

Another principal candidate regulating ovarian blood vessel formation is VEGFA, which acts through three tyrosine kinase family receptors (Ferrara \& Davis-Smyth 1997). Several studies have established that VEGFA has an undisputed role in the regulation of ovarian angiogenesis (Phillips et al. 1990, Ravindranath et al. 1992, Koos 1995, Redmer et al. 2001). In our study, both in immature and adult rats, staining for VEGFA and KDR was greater in POF than in PF and EAF. These results are consistent with previous data observed by other authors by measurement of Vegfa mRNA in several species. It has been demonstrated in bovines and porcines that the expression of Vegfa mRNA is weak during early follicular development and enhanced during dominant follicle development (Barboni et al. 2000, Greenaway et al. 2004). Similar results have been found in rat ovary (Maisonpierre et al. 1997). Moreover, Taylor et al. (2004) have established in primates that Vegfa mRNA is absent in primordial, primary, and early secondary follicles and it is first detectable in theca and Gc layers of secondary follicles when the follicle starts to develop its own vasculature. However, Redmer et al. (2001) have observed in sheep that VEGFA protein is localized exclusively to the theca layer of POF, and that the granulosa was devoid of staining. These variations in VEGFA expression could be due to the different species involved.

Further evidence for the role of VEGFA in the regulation of ovarian follicular angiogenesis is derived from functional studies. For example, the administration of substances that inactivate VEGFA blocks the development and function of POF as demonstrated by analysis of 

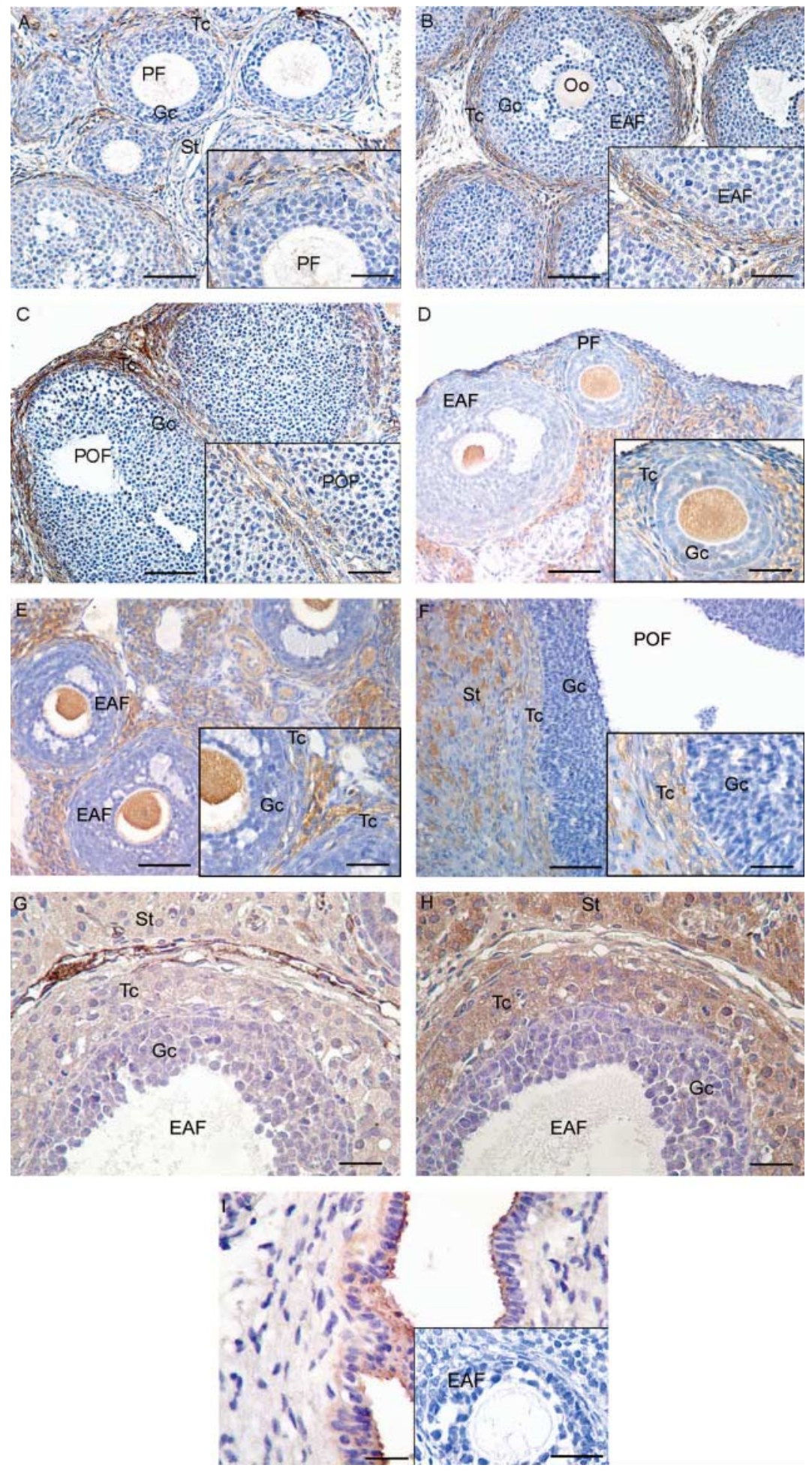

Figure 5 Immunolocalization of TEK and von Willebrand factor in rat ovarian follicles at different stages of development. (A) Untreated prepubertal rats. (B) DES-treated prepubertal rats. (C) eCG-treated prepubertal rats. D-F shows representative fields of ovarian sections from postpubertal cycling rats. ( $\mathrm{G}$ and $\mathrm{H}$ ) Immunohistochemical staining for von Willebrand (marker of vascular endothelial cells) and TEK was performed on adjacent paraffin sections. (I) Human endometrium was used as a positive control and the glandular epithelium showed immunoreactivity for TEK. The negative control was carried out in the absence of primary antibody (inset). PF, preantral follicle; EAF, early antral follicle; POF, preovulatory follicle; Gc, granulosa cells; Tc, theca cells; Oo, oocyte; St, stroma. Scale bars represent (A-F and negative control) $50 \mu \mathrm{m}$; (G-I and insets) $20 \mu \mathrm{m}$. ovarian histology (Maisonpierre et al. 1997, Wulff et al. 2001) or hormone measurements (Zimmermann et al. 2001). In addition, we showed that the local inhibition of VEGFA activity appears to produce an increase in ovarian apoptosis through an imbalance among the
BCL2 family members, thus leading a larger number of follicles to atresia (Abramovich et al. 2006).

Despite the increasing number of reports suggesting that VEGFA is involved in vascular development during folliculogenesis, little is known about the functional role 

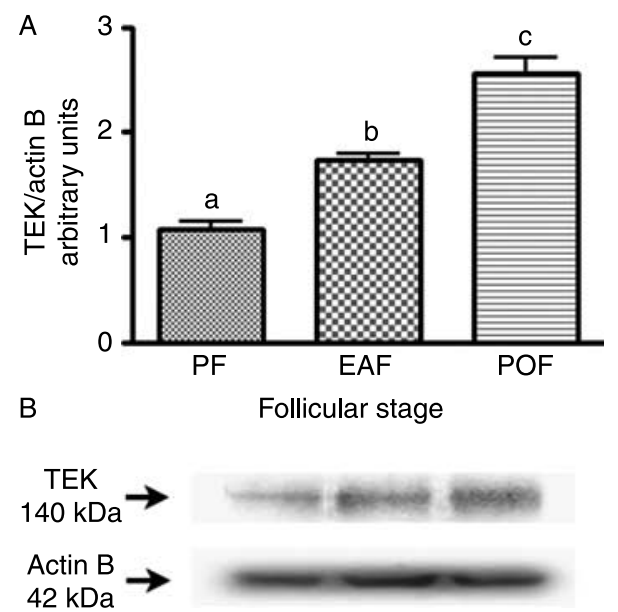

Figure 6 Analysis of the expression of TEK on follicular development in ovaries from prepubertal, DES-treated or superovulated rats. (A) Densitometric quantification of TEK content. (B) Representative immunoblot of TEK. Bars represent the mean \pm s.E.M. normalized to $\beta$-actin. Different letters over the bars mean significant differences between groups $(n=8 ; P<0.05)$.

of ANGPTs in the ovary (Hazzard et al. 2000, Wulff et al. 2000, Hata et al. 2002). Direct evidence for the role of angiopoietins in the ovary comes from observations in rhesus monkeys (Xu et al. 2005) and rats (Parborell et al. 2008). Xu et al. (2005) have demonstrated that local injection of ANGPT2 into the POF is able to block ovulation, while the same dose of ANGPT1 is not. It has been proposed that ANGPT2 inhibits the action of ANGPT1 at the level of their common receptor and that the correct balance between these factors is essential for maintaining the integrity of the POF. In this regard, in the presence of VEGFA, increased expression of ANGPT2 is associated with angiogenesis, while in the absence of VEGFA or other angiogenic factors; its expression is associated with degenerative changes in the vasculature.

We have previously shown that the inhibition of ANGPT1 activity causes an increase in the number of atretic follicles mediated by ovarian apoptosis through an imbalance in the ratio of antiapoptotic: proapoptotic proteins (Parborell et al. 2008) and it has been suggested that a possible paracrine effect would take place on Gcs, which may be mediated by the TEK receptor in Tc. In agreement with this, in this study we observed that ANGPT1 protein and its receptor TEK are only present in theca and endothelial cells during follicular development. This leads us to think that in developing follicles ANGPT1 may act as not only a proangiogenic factor but also a cell survival factor.

In conclusion, this study shows that the protein expression of ANGPT1, ANGPT2, their receptor TEK, as well as of VEGFA and its receptor KDR, clearly increased during rat follicular development. These findings suggest that this angiogenic system is involved in the functional changes that occur during follicular development.

\section{Materials and Methods \\ Materials and reagents}

eCG (Novormon) was provided by Syntex S.A. (Buenos Aires, Argentina). Primary antibodies for VEGFA (sc-152), ANGPT1 (sc-9360), ANGPT2 (sc-7017), KDR (sc-6251), and TEK (sc9062) were purchased from Santa Cruz Biotechnology Inc. (Santa Cruz, CA, USA). Primary antibodies for Von Willebrand (A0082) were from Dako Cytomation (Dako Denmark, DK2600, Denmark) and actin B antibody was from Abcam (ab6276; Cambridge, Science Park, UK). Anti-rabbit, anti-mouse, and anti-goat secondary antibodies conjugated with HRP, HEPES, and SDS were purchased from Sigma Chemical Co. (DMEM, $4.5 \mathrm{~g}$ glucose/l), Ham F-12 nutrient mixture (F12), fungizone $(250 \mathrm{mg} / \mathrm{ml})$, and gentamicin $(10 \mathrm{mg} / \mathrm{ml})$ were from Gibco Laboratories. All other chemicals were of reagent grade and were obtained from standard commercial sources.

\section{Animal preparation}

General care and housing of rats were carried out at the Instituto de Biología y Medicina Experimental (IByME), Buenos Aires, Argentina. Rats ( $n=8$ /group for each treatment) were from our colony. Immature and adult female Sprague-Dawley rats (21-23 days old and 60 days old) were allowed access to food and water ad libitum and kept at room temperature $\left(21-23^{\circ} \mathrm{C}\right)$ on a $12 \mathrm{~h}$ light: $12 \mathrm{~h}$ darkness cycle.

To obtain follicles at different developmental stages for immunochemistry and western immunoblot analyses, immature rats were injected s.c. either with DES ( $1 \mathrm{mg} / \mathrm{rat})$ dissolved in corn oil daily for 3 days to stimulate the development of EAF or with eCG (25 IU/rat, also known as PMSG) for 2 days to induce the development of POF; Li et al. 1998). To obtain PF, no treatment was applied to the immature rats.

To confirm that the differences observed in the expression of angiogenic factors and their receptors are due to the follicular developmental stage and not a consequence of differences in the hormonal treatment, untreated adult rats were used. Estrous cycle stages were determined by daily examination of vaginal cytology. Ovaries were collected in proestrus. Rats were then killed by $\mathrm{CO}_{2}$ asphyxiation and ovaries were removed and cleaned of adhering tissue in culture medium. One ovary per immature rat was used for western immunoblot assays and the contralateral ovary for immunohistochemical assays. Two ovaries per adult rat were used for immunohistochemical assays.

The experimental protocols were approved by the Animal Experimentation Committee of the IByME.

\section{Follicle isolation for western immunoblot}

For isolation of PF, we used a method previously described by Flaws et al. (1994). Briefly, ovaries obtained from prepubertal rats were minced using 26G1/2 syringe needles. Minced tissue 

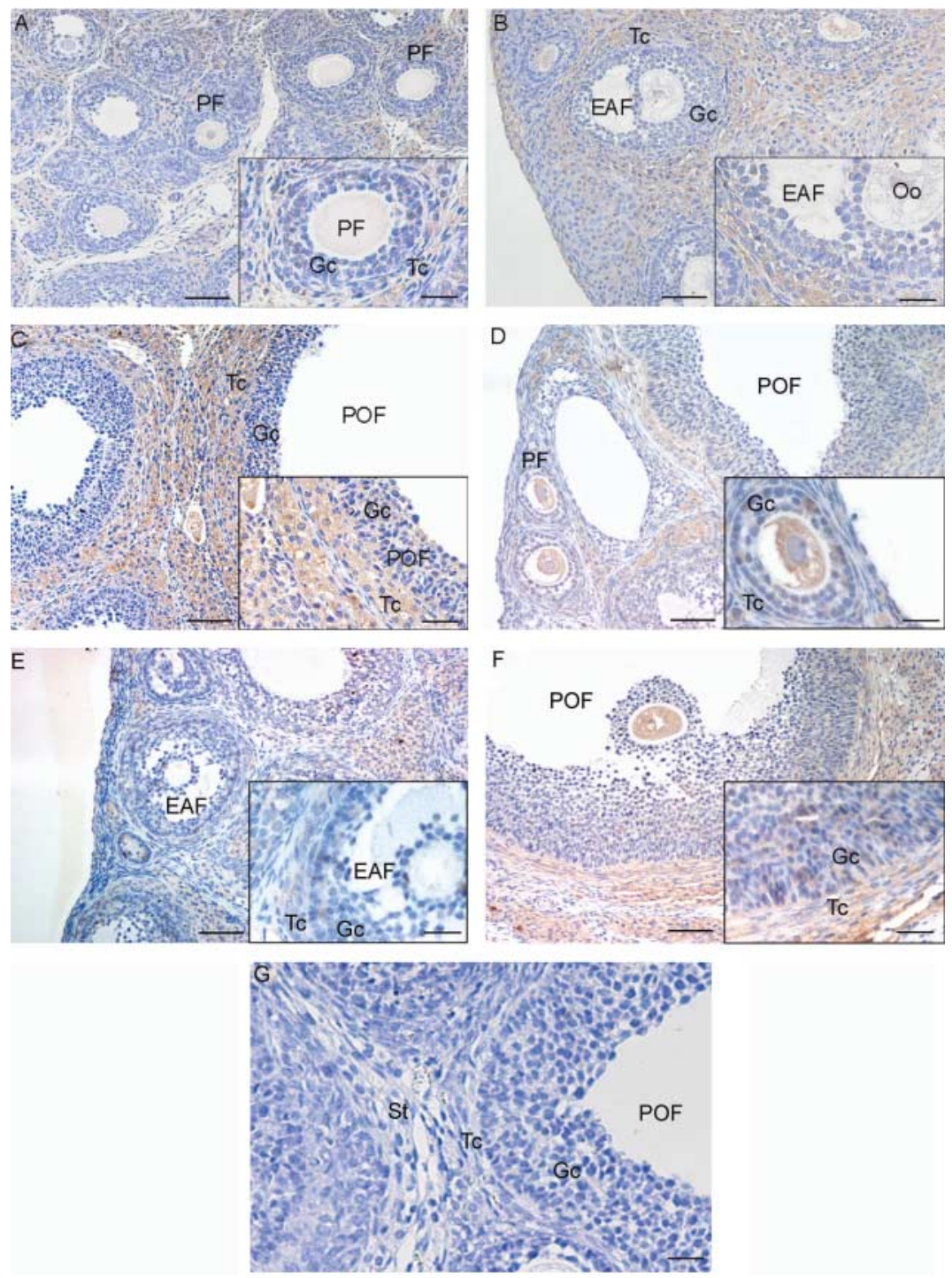

Figure 7 Immunohistochemical staining for VEGF in rat ovarian follicles at different developmental stages. (A) Untreated prepubertal rats. (B) DEStreated prepubertal rats. (C) eCG-treated prepubertal rats. D-F shows representative fields of ovarian sections from postpubertal cycling rats; (G) Negative control. Specificity for VEGFA is supported by the loss cytoplasmic staining in follicular tissue preabsorbed with VEGFA peptide or in the absence of the primary antibody (not shown). PF, preantral follicle; EAF, early antral follicle; POF, preovulatory follicle; Gc, granulosa cells; Tc, theca cells; Oo, oocyte; St, stroma. Scale bars represent (A-F) $50 \mu \mathrm{m}$; (G and insets) $20 \mu \mathrm{m}$. was dissociated $\left(20 \mathrm{~min}\right.$ in a water shaking bath at $37^{\circ} \mathrm{C}$ ) in $2.5 \mathrm{ml}$ HEPES containing collagenase (4800 units; $27.2 \mathrm{mg} / \mathrm{ml}$ ), DNase (1910 units; $10 \mathrm{mg} / \mathrm{ml}$; D4527, Sigma), and $1 \%$ BSA (A7888, Sigma). The dissociated tissue was then washed three times in HEPES and passed through a nylon filter with $210 \mu \mathrm{m}$ pores (Small Parts CMN-210, Miramar, FL, USA). All excluded structures (follicles $>210 \mu \mathrm{m}$ in diameter and corpora lutea) were discarded and follicles smaller than $210 \mu \mathrm{m}$ were considered PF. Ovaries from DES-treated rats were used to obtain EAF, whereas ovaries from eCG-superovulated rats were used to obtain POF. In both cases, individual ovarian follicles were dissected from the ovary under a stereoscopic microscope as described previously (Parborell et al. 2002). Briefly, follicles (EAF: 300-400 $\mu \mathrm{m}$, POF: $>450 \mu \mathrm{m}$ in diameter) from eight ovaries per group were frozen and used for western immunoblot. From each ovary, a pool of isolated follicles (50 follicles/ovary) was frozen, and the results obtained from each pool were considered a single datum.

\section{Immunohistochemistry}

Sections $(4 \mu \mathrm{m})$ of formalin-fixed paraffin-embedded tissue were deparaffinized in xylene and hydrated in graded ethanols. Sections were treated for 30 min with $3 \% \mathrm{H}_{2} \mathrm{O}_{2}$ in PBS, followed by successive PBS washes. When studying the localization of ANGPT1 and ANGPT2 proteins, the slides were heated in a microwave oven in a citric acid buffer $0.01 \mathrm{M}(\mathrm{pH}=6)$ for $9 \mathrm{~min}$ at $600 \mathrm{~W}$ followed by $20 \mathrm{~min}$ off. For VEGFA and Von Willebrand (marker for endothelial cells) proteins, the slides were exposed to proteinase $\mathrm{K}$ in PBS $(20 \mu \mathrm{g} / \mathrm{ml})$ for $15 \mathrm{~min}$, followed by two successive washes with distilled water. For KDR and TEK proteins, there was no antigenic retrieval treatment. Then, sections were blocked with $2 \%$ BSA for $20 \mathrm{~min}$ and incubated overnight with an antibody directed against the corresponding peptide overnight at $4{ }^{\circ} \mathrm{C}$ in a humidified chamber (anti ANGPT1 1/50, anti ANGPT2 1/50, anti TEK 1/50, anti VEGFA 1/100, anti KDR 1/100, anti Von Willebrand $1 / 100$ in PBS). The sections 


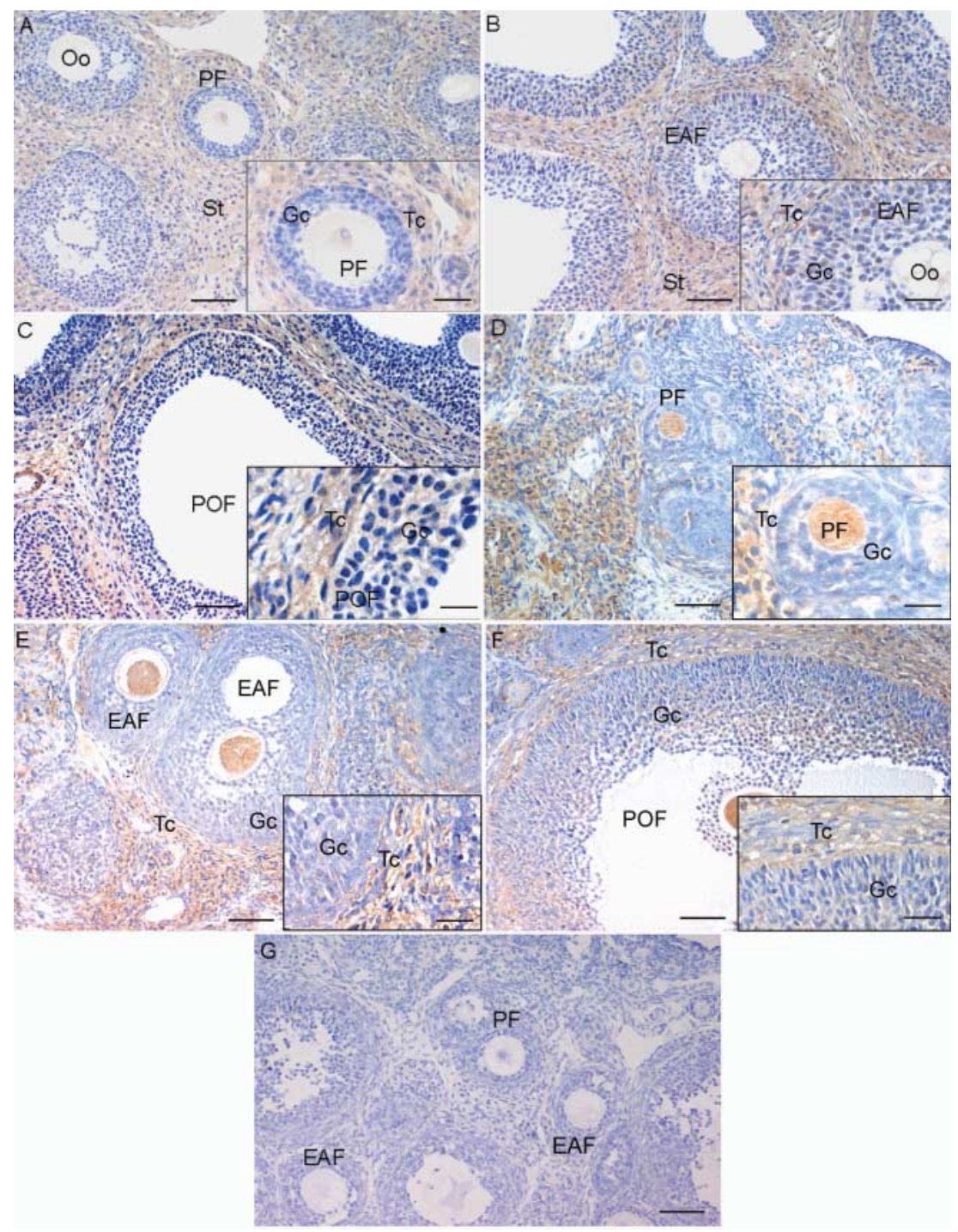

Figure 8 Immunohistochemical staining for KDR during follicular development in the rat ovary. (A) Untreated prepubertal rats. (B) DES-treated prepubertal rats. (C) eCG-treated prepubertal rats. D-F shows representative fields of ovarian sections from postpubertal cycling rats.

(G) Negative control was carried out in the absence of primary antibody. PF, preantral follicle; EAF, early antral follicle; POF, preovulatory follicle; Gc, granulosa cells; Tc, theca cells; Oo, oocyte; St, stroma. Scale bars represent (A-G) $50 \mu \mathrm{m}$; (insets) $20 \mu \mathrm{m}$. were washed in PBS. ANGPT1 and ANGPT2 were detected with a biotin-conjugated rabbit anti-goat IgG (room temperature, 30 min, 1/400 in PBS; Vector Laboratories, Burlingame, CA, USA). TEK, VEGFA, and Von Willebrand were detected with a biotin-conjugated goat anti-rabbit IgG (RT, $30 \mathrm{~min}$, 1/400 in PBS; Vector Laboratories). KDR was detected with a biotin-conjugated goat anti-mouse IgG (RT, $30 \mathrm{~min}, 1 / 400$ in PBS; Vector Laboratories). After additional PBS washes, sections were incubated with an avidin-peroxidase complex (Vector Laboratories) for $30 \mathrm{~min}$ in a humidified chamber, washed in PBS, and treated with 3,5-diaminobenzide tetrahydrochloride $(0.05 \%, \mathrm{vol} / \mathrm{vol})$. The appearance of brown reaction product was observed by light microscopy. Sections were washed in PBS and counterstained with hematoxylin, dehydrated in graded alcohols, and mounted. Negative controls were obtained in the absence of primary antibody, or by preabsorbing the antibodies with an excess of
ANGPT1-, ANGPT2-, and VEGFA-blocking peptides (sc9360P, sc-7017P and sc-152P; Santa Cruz Biotechnology) respectively; no specific immunoreactivity was detected in these sections.

Morphological characteristics of PF include their size (PF: 120-200 $\mu \mathrm{m}$ ) and an oocyte surrounded by two to four layers of Gc. EAF are defined as follicles of 300-400 $\mu \mathrm{m}$ in diameter with a small antral cavity and a thin theca layer. Finally, features of preovulatory antral follicles (POF) include their size ( $>450 \mu \mathrm{m}$ in diameter), a well-defined and thick granulosa layer and a displaced-acentrically oocyte by the developing antrum (Hirshfield \& Midgley 1978, Hughes \& Gorospe 1991).

\section{Western immunoblot}

Fifty follicles per ovary were resuspended in $350 \mu$ lysis buffer (20 mM Tris- $\mathrm{HCl}(\mathrm{pH} 8), 137 \mathrm{mM} \mathrm{NaCl}, 1 \% \mathrm{NP}-40$, and $10 \%$ 


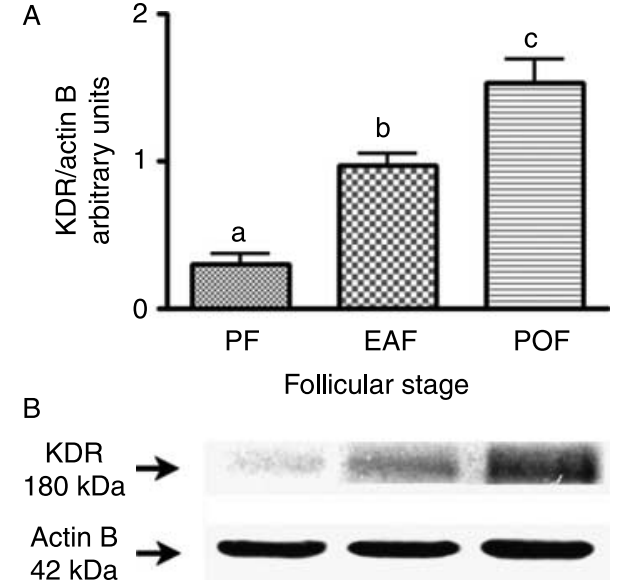

Figure 9 Analysis of the expression of KDR on follicular development in ovaries from prepubertal, DES-treated or superovulated rats. (A) Densitometric quantification of KDR content. (B) Representative immunoblot of KDR. Bars represent the mean \pm S.E.M. normalized to $\beta$-actin. Different letters over the bars mean significant differences between groups $(n=8 ; P<0.05)$.

glycerol) supplemented with protease inhibitors $(0.5 \mathrm{mM}$ PMSF, $0.025 \mathrm{mM} \mathrm{N-CBZ-L-phenylalanine} \mathrm{chloromethyl}$ ketone, $0.025 \mathrm{mM} \mathrm{N}$-p-tosyl-lysine chloromethyl ketone, and 0.025 mM-L-1-tosylamide-2-phenyl-ethylchloromethyl ketone; all the products were obtained from Sigma) and homogenized with an Ultra-Turrax (IKA Werk, Breisgau, Germany) homogenizer. Samples were centrifuged at $4{ }^{\circ} \mathrm{C}$ for $10 \mathrm{~min}$ at $10000 \mathrm{~g}$, and the resulting pellets discarded. Protein concentration in the supernatant was measured by the Bradford assay. After boiling for $5 \mathrm{~min}, 60 \mu \mathrm{g}$ protein was applied to an $8-15 \%$ SDS-polyacrylamide gel (ANGPT1 and ANGPT2: 15\%; TEK and KDR: $8 \%$ ), and electrophoresis was performed at $25 \mathrm{~mA}$ for $1.5 \mathrm{~h}$. The resolved proteins were transferred for $2 \mathrm{~h}$ onto nitrocellulose membranes (Bio-Rad). The blot was preincubated in blocking buffer (5\% non-fat milk and 0.05\% Tween-20 in $20 \mathrm{mM}$ triethanolamine-buffered saline (TBS; $\mathrm{pH}$ 8.0) for $1 \mathrm{~h}$ at room temperature and incubated with appropriate primary antibodies (anti VEGFA 1/200, ANGPT1 1/500, anti ANGPT2 1/100, anti TEK 1/50, anti KDR 1/50, anti von Willebrand $1 / 100$, and anti actin B 1/10 000) in $0.05 \%$ Tween-20 in TBS overnight at $4{ }^{\circ} \mathrm{C}$. The blot was then incubated with anti-rabbit, anti-mouse, or anti-goat secondary antibodies conjugated with $\operatorname{HRP}(1: 1000)$ and finally detected by chemiluminescence and autoradiography with an X-ray film. Negative controls were obtained in the absence of the primary antibody.

\section{Quantification for the western immunoblot}

In each experiment, equal amounts of protein were loaded for all samples, and protein extracts from PF, EAF, and POF were loaded on the same gel. For quantification, a screening was performed on blots with an X-ray film using different times of exposure to optimize the signal. The density of each band was normalized to the density of the actin B band that was used as an internal control. The levels of protein were compared and analyzed with densitometric studies by Scion Image for Windows (Scion Corporation, NIH, Washington, DC, USA). Optical density data are expressed as arbitrary units \pm S.E.M. $(n=8)$.

\section{Data analysis}

Data are expressed as the mean \pm s.E.M. of three to five experiments. Representative gels are shown in the figures. Statistical analysis was performed by ANOVA, followed by the Tukey test or the multiple comparisons Newman-Keuls test. Values of $P<0.05$ were considered significant.
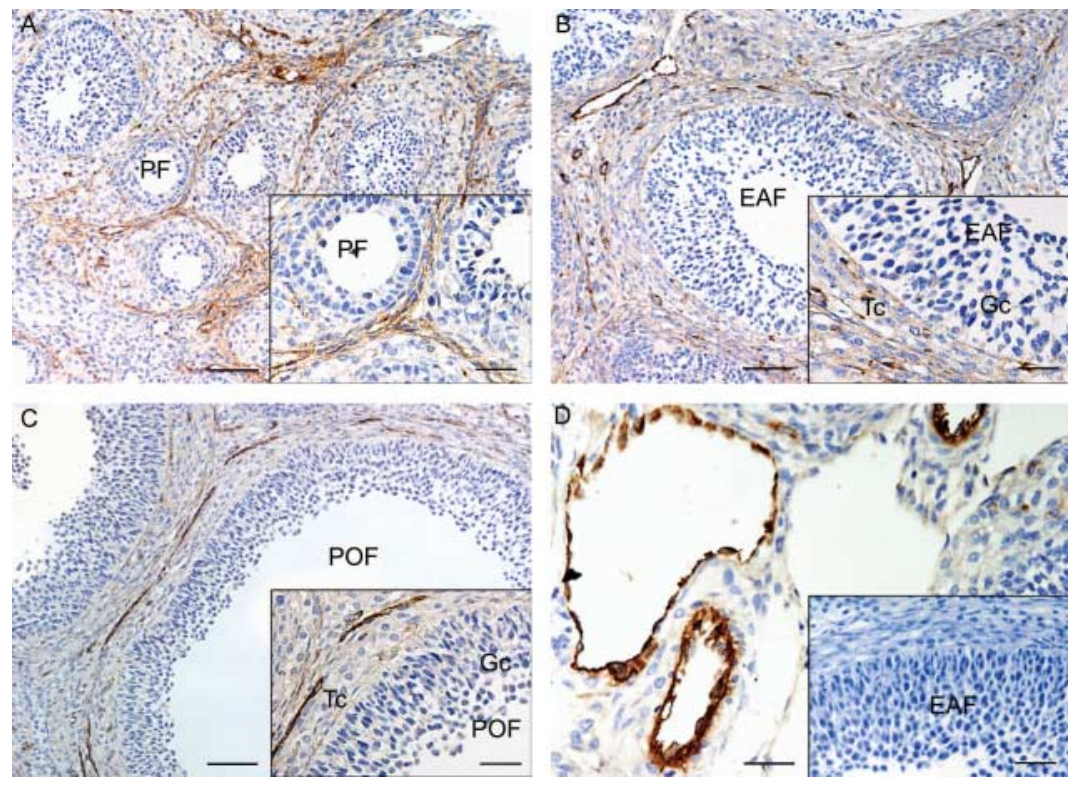

Figure 10 von Willebrand factor immunostaining performed on different follicular stages in the rat ovary. (A-D) shows representative fields of ovarian sections enriched in (A) Preantral follicles (PF); (B) Early antral follicles (EAF) or (C) Preovulatory follicles (POF); (D) Endothelial cells from blood vessels were used as a positive control. The inset in (D) shows the negative control that was carried out in the absence of primary antibody. Gc, granulosa cells; Tc, theca cells; Oo, oocyte; St, stroma; and Ec, endothelial cell. Scale bars represent (A-C) $50 \mu \mathrm{m}$; (D and insets) $20 \mu \mathrm{m}$. 


\section{Declaration of interest}

The authors declare that there is no conflict of interest that could be perceived as prejudicing the impartiality of the research reported.

\section{Funding}

This study was supported by ANPCyT (PICT 5-26047), CONICET (PIP5471), and Roemmers Foundation.

\section{Acknowledgements}

We thank Pablo Do Campo and Diana Bas (IByME-CONICET) for their technical assistance.

\section{References}

Abramovich D, Parborell F \& Tesone M 2006 Effect of a vascular endothelial growth factor (VEGF) inhibitory treatment on the folliculogenesis and ovarian apoptosis in gonadotropin-treated prepubertal rats. Biology of Reproduction 75 434-441.

Barboni B, Turriani M, Galeati G, Spinaci M, Bacci ML, Forni M \& Mattioli M 2000 Vascular endothelial growth factor production in growing pig antral follicles. Biology of Reproduction 63 858-864.

Celik-Ozenci C, Akkoyunlu G, Kayisli UA, Arici A \& Demir R 2003 Localization of vascular endothelial growth factor in the zona pellucida of developing ovarian follicles in the rat: a possible role in destiny of follicles. Histochemistry and Cell Biology 120 383-390.

Davis S, Aldrich TH, Jones PF, Acheson A, Compton DL, Jain V, Ryan TE, Bruno J, Radziejewski C, Maisonpierre PC et al. 1996 Isolation of angiopoietin-1, a ligand for the TIE2 receptor, by secretion-trap expression cloning. Cell 87 1161-1169.

Ferrara N 1999 Molecular and biological properties of vascular endothelial growth factor. Journal of Molecular Medicine 77 527-543.

Ferrara N \& Davis-Smyth T 1997 The biology of vascular endothelial growth factor. Endocrine Reviews 18 4-25.

Flaws JA, Doerr JK, Sipes IG \& Hoyer PB 1994 Destruction of preantral follicles in adult rats by 4-vinyl-1-cyclohexene diepoxide. Reproductive Toxicology 8 509-514.

Folkman J \& Klagsbrun M 1987 Angiogenic factors. Science 235 442-447.

Geva E \& Jaffe RB 2000 Role of vascular endothelial growth factor in ovarian physiology and pathology. Fertility and Sterility 74 429-438.

Greenaway J, Connor K, Pedersen HG, Coomber BL, LaMarre J \& Petrik J 2004 Vascular endothelial growth factor and its receptor, Flk-1/KDR, are cytoprotective in the extravascular compartment of the ovarian follicle. Endocrinology 145 2896-2905.

Greenwald GS 1989 Temporal and topographic changes in DNA synthesis after induced follicular atresia. Biology of Reproduction 41 175-181.

Hanahan D 1997 Signaling vascular morphogenesis and maintenance. Science 277 48-50.

Hata K, Udagawa J, Fujiwaki R, Nakayama K, Otani H \& Miyazaki K 2002 Expression of angiopoietin-1, angiopoietin-2, and Tie2 genes in normal ovary with corpus luteum and in ovarian cancer. Oncology 62 340-348.

Hazzard TM, Molskness TA, Chaffin CL \& Stouffer RL 1999 Vascular endothelial growth factor (VEGF) and angiopoietin regulation by gonadotrophin and steroids in macaque granulosa cells during the peri-ovulatory interval. Molecular Human Reproduction 5 1115-1121.

Hazzard TM, Christenson LK \& Stouffer RL 2000 Changes in expression of vascular endothelial growth factor and angiopoietin-1 and -2 in the macaque corpus luteum during the menstrual cycle. Molecular Human Reproduction 6 993-998.

Hirchenhain J, Huse I, Hess A, Bielfeld P, De Bruyne F \& Krussel JS 2003 Differential expression of angiopoietins 1 and 2 and their receptor Tie-2 in human endometrium. Molecular Human Reproduction 9 663-669.

Hirshfield AN \& Midgley AR Jr 1978 Morphometric analysis of follicular development in the rat. Biology of Reproduction 19 597-605.
Hughes FM Jr \& Gorospe WC 1991 Biochemical identification of apoptosis (programmed cell death) in granulosa cells: evidence for a potential mechanism underlying follicular atresia. Endocrinology 129 2415-2422.

Klagsbrun M \& D'Amore PA 1996 Vascular endothelial growth factor and its receptors. Cytokine and Growth Factor Reviews 7 259-270.

Koos RD 1995 Increased expression of vascular endothelial growth/permeability factor in the rat ovary following an ovulatory gonadotropin stimulus: potential roles in follicle rupture. Biology of Reproduction 52 1426-1435.

Li J, Kim JM, Liston P, Li M, Miyazaki T, Mackenzie AE, Korneluk RG \& Tsang BK 1998 Expression of inhibitor of apoptosis proteins (IAPs) in rat granulosa cells during ovarian follicular development and atresia. Endocrinology 139 1321-1328.

Maisonpierre PC, Suri C, Jones PF, Bartunkova S, Wiegand SJ, Radziejewski C, Compton D, McClain J, Aldrich TH, Papadopoulos N et al. 1997 Angiopoietin-2, a natural antagonist for Tie2 that disrupts in vivo angiogenesis. Science 277 55-60.

Pandya NM, Dhalla NS \& Santani DD 2006 Angiogenesis - a new target for future therapy. Vascular Pharmacology 44 265-274.

Parborell F, Pecci A, Gonzalez O, Vitale A \& Tesone M 2002 Effects of a gonadotropin-releasing hormone agonist on rat ovarian follicle apoptosis: regulation by EGF and the expression of $\mathrm{Bcl}-2$-related genes. Biology of Reproduction 67 481-486.

Parborell F, Abramovich D \& Tesone M 2008 Intrabursal administration of the antiangiopoietin 1 antibody produces a delay in rat follicular development associated with an increase in ovarian apoptosis mediated by changes in the expression of BCL2 related genes. Biology of Reproduction 78 506-513.

Phillips HS, Hains J, Leung DW \& Ferrara N 1990 Vascular endothelial growth factor is expressed in rat corpus luteum. Endocrinology 127 965-967.

Ravindranath N, Little-Ihrig L, Phillips HS, Ferrara N \& Zeleznik AJ 1992 Vascular endothelial growth factor messenger ribonucleic acid expression in the primate ovary. Endocrinology 131 254-260.

Redmer DA, Doraiswamy V, Bortnem BJ, Fisher K, Jablonka-Shariff A, Grazul-Bilska AT \& Reynolds LP 2001 Evidence for a role of capillary pericytes in vascular growth of the developing ovine corpus luteum. Biology of Reproduction 65 879-889.

Scharpfenecker M, Fiedler U, Reiss Y \& Augustin HG 2005 The Tie-2 ligand angiopoietin-2 destabilizes quiescent endothelium through an internal autocrine loop mechanism. Journal of Cell Science 118 771-780.

Shimizu T, Jiang JY, lijima K, Miyabayashi K, Ogawa Y, Sasada H \& Sato E 2003 Induction of follicular development by direct single injection of vascular endothelial growth factor gene fragments into the ovary of miniature gilts. Biology of Reproduction 69 1388-1393.

Shweiki D, Itin A, Neufeld G, Gitay-Goren H \& Keshet E 1993 Patterns of expression of vascular endothelial growth factor (VEGF) and VEGF receptors in mice suggest a role in hormonally regulated angiogenesis. Journal of Clinical Investigation 91 2235-2243.

Stouffer RL, Martinez-Chequer JC, Molskness TA, Xu F \& Hazzard TM 2001 Regulation and action of angiogenic factors in the primate ovary. Archives of Medical Research 32 567-575.

Suri C, Jones PF, Patan S, Bartunkova S, Maisonpierre PC, Davis S, Sato TN \& Yancopoulos GD 1996 Requisite role of angiopoietin-1, a ligand for the TIE2 receptor, during embryonic angiogenesis. Cell 87 1171-1180.

Tanaka J, Acosta TJ, Berisha B, Tetsuka M, Matsui M, Kobayashi S, Schams D \& Miyamoto A 2004 Relative changes in mRNA expression of angiopoietins and receptors tie in bovine corpus luteum during estrous cycle and prostaglandin F2alpha-induced luteolysis: a possible mechanism for the initiation of luteal regression. Journal of Reproduction and Development $\mathbf{5 0} 619-626$.

Taylor PD, Hillier SG \& Fraser HM 2004 Effects of GnRH antagonist treatment on follicular development and angiogenesis in the primate ovary. Journal of Endocrinology 183 1-17.

Wulff C, Wilson H, Largue P, Duncan WC, Armstrong DG \& Fraser HM 2000 Angiogenesis in the human corpus luteum: localization and changes in angiopoietins, tie-2, and vascular endothelial growth factor messenger ribonucleic acid. Journal of Clinical Endocrinology and Metabolism 85 4302-4309. 
Wulff C, Wiegand SJ, Saunders PT, Scobie GA \& Fraser HM 2001 Angiogenesis during follicular development in the primate and its inhibition by treatment with truncated Flt-1-FC (vascular endothelial growth factor Trap(A40)). Endocrinology 142 3244-3254.

Xu F, Hazzard TM, Evans A, Charnock-Jones S, Smith S \& Stouffer RL 2005 Intraovarian actions of anti-angiogenic agents disrupt periovulatory events during the menstrual cycle in monkeys. Contraception $\mathbf{7 1}$ 239-248.

Zeleznik AJ, Schuler HM \& Reichert LE Jr 1981 Gonadotropin-binding sites in the rhesus monkey ovary: role of the vasculature in the selective distribution of human chorionic gonadotropin to the preovulatory follicle. Endocrinology 109 356-362.
Zimmermann RC, Xiao E, Husami N, Sauer MV, Lobo R, Kitajewski J \& Ferin M 2001 Short-term administration of antivascular endothelial growth factor antibody in the late follicular phase delays follicular development in the rhesus monkey. Journal of Clinical Endocrinology and Metabolism 86 768-772.

Received 19 March 2008

First decision 23 April 2008

Revised manuscript received 29 October 2008

Accepted 7 November 2008 